\title{
LA VIABILIDAD Y SOSTENIBILIDAD DE LAS EXPERIENCIAS DE TRABAJO ASOCIATIVO Y AUTOGESTIONADO DESDE UNA PERSPECTIVA PLURAL
}

Gonzalo Vázquez ${ }^{1}$

\section{Resumen/Abstract}

En este artículo se plantean una serie de reflexiones y aportes sobre la problemática de la viabilidad o sostenibilidad de las experiencias de trabajo asociativo y autogestionado que se desarrollan en Argentina y otros países de la región. No se trata de un trabajo de evaluación empírica de esta realidad emergente, sino más bien un texto reflexivo y conceptual. En general, el artículo aporta argumentos para repensar la cuestión de la sostenibilidad de estas experiencias asumiendo una perspectiva económica crítica y plural. ${ }^{2}$

Palabras clave: emprendimientos asociativos, trabajo autogestionado, sostenibilidad, economía social y solidaria

THE VIABILITY AND SUSTAINABILITY OF WORK ASSOCIATIVE AND SELF-MANAGED EXPERIENCES FROM A PLURAL PERSPECTIVE

This article presents a number of reflections and contributions on the issue of the viability and sustainability of the experiences of associative and self-managed work developed in Argentina and other countries in the region. It is not an empirical evaluation of this emerging reality, but rather a reflective and conceptual text. In general, the article provides arguments to rethink the question of the sustainability of these experiences, assuming a critic and plural economic perspective.

Keywords: associative enterprises, self-managed work, sustainability, social and solidarity economy.

1 Argentino, Universidad Nacional de General Sarmiento y Universidad de Luján. E-mail: gvazquez@ungs.edu.ar

2 Este artículo es una versión revisada y aumentada de la ponencia "Viabilidad y sostenibilidad de las experiencias asociativas", presentada el miércoles 27 de mayo de 2015 en la Universidad Academia de Humanismo Cristiano de Santiago (Chile), en el marco del Seminario Internacional de Econo-mía Social y Solidaria Más allá del neoliberalismo. 


\section{Introducción}

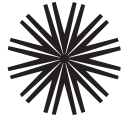

En el presente artículo planteo una serie de reflexiones y aportes acerca de la problemática de la sostenibilidad de los emprendimientos asociativos de trabajadores autogestionados. Lo hago asumiendo distintos tonos y niveles de profundidad, y para ello organizo el texto en tres partes: en la parte I comparto -en un lenguaje no demasiado académicoalgunas ideas y preguntas acerca de la realidad emergente de las nuevas experiencias de trabajo asociativo y autogestionado; en la parte II planteo -de manera más o menos exploratoria- algunas conceptualizaciones sobre este tipo de emprendimientos y sobre las ideas de viabilidad y sostenibilidad; en la parte III presento una breve sistematización del debate académico latinoamericano acerca de cómo mejorar las condiciones de sostenibilidad/viabilidad de los emprendimientos de trabajadores autogestionados dentro del campo de la Economía Social y Solidaria (ESS).

Si bien el trabajo se apoya en estudios y conocimientos previos de la realidad concreta de los emprendimientos de trabajadores autogestionados en Argentina, éste no es artículo en donde se realice una evaluación empírica de estas experiencias emergentes, sino más bien un texto reflexivo, exploratorio y conceptual. En general, considero que el artículo aporta diversos argumentos para repensar la cuestión de la sostenibilidad de estas experiencias asumiendo una perspectiva económica crítica, plural y contrahegemónica. Mi punto de vista está claramente influenciado por la realidad en la que desarrollo mi trabajo (en una universidad pública de la periferia de Buenos Aires, Argentina) y por ello no pretendo que mis reflexiones y aportes sean válidos para otras realidades nacionales o sectoriales, aunque seguramente existan puntos de encuentro y cuestiones en común, sobre todo con los otros países sudamericanos.

\section{Parte I. Problematizando la cuestión de la sostenibilidad de las experiencias de trabajo asociativo y autogestionado desde una mirada crítica del sentido común dominante}

\section{I.1. Experiencias de trabajadores que buscan alternativas}

Una gran cantidad de trabajadores, ante la pérdida o la dificultad de 
conseguir un empleo asalariado, o buscando una experiencia de autonomía frente al capital, se dan como estrategia alternativa la conformación de emprendimientos productivos o comerciales autogestionados, o sea buscan organizar su propio emprendimiento para obtener ingresos que les permitan vivir dignamente de su trabajo. Muchos trabajadores encaran este desafío individualmente, aunque siempre apoyados por el trabajo de parientes y allegados. Y muchos otros lo hacen asociativa y solidariamente con otros trabajadores, compañeros en sus intentos y dificultades. En cualquier caso, el desafío es enorme: lograr que esta actividad autogestionada se convierta en "su trabajo", con el cual satisfacer sus necesidades cotidianas.

En general, estos emprendimientos asociativos de trabajadores autogestionados son experiencias grupales en las que el trabajo y la cooperación ocupan el lugar central; en las que se producen bienes o servicios destinados a su venta para generar ingresos en dinero; en donde las decisiones se toman de manera relativamente participativa y democrática; y cuyo fin y sentido último es la reproducción de la vida de sus integrantes y de sus familias, es decir que están orientados hacia la satisfacción de sus necesidades y no hacia la maximización de ganancias para acumular capital. Ejemplos de este tipo de experiencias pueden ser los emprendimientos asociativos barriales (productivos, comerciales o de servicios) que impulsan un conjunto de vecinos buscando dar respuesta a necesidades y demandas del propio barrio en el que viven; o las cooperativas de trabajo impulsadas por diversos gupos de trabajadores o promovidas por ONGs o por el estado; o las empresas recuperadas por sus trabajadores luego de una quiebra o abandono del patrón entre otros emprendimientos que podemos observar cada vez más frecuentemente en nuestros países.

Ahora bien, sabemos que muchos de estos emprendimientos enfrentan variadas dificultades para sostenerse a lo largo del tiempo: falta de recursos para la producción, financiamiento inadecuado e insuficiente, acceso a insumos relativamente caros, dificultades en la comercialización, falta de capacidades para la gestión, conflictos internos, problemas legales y contables, precariedad laboral, sobrecarga de trabajo e ingresos insuficientes... sólo para mencionar los problemas más comunes y generales. (Abramovich y Vázquez, 2007; Gaiger, 2004; Coraggio y Federico Sabaté, 2010)

Entonces surgen en cada experiencia, inevitables, las preguntas acerca de su viabilidad o sostenibilidad: ¿será sostenible este emprendimiento? ¿será viable este proyecto de trabajo? ¿bajo qué condiciones? ¿con qué tipo de ayudas y por cuánto tiempo? 


\section{I.2. La autosostenibilidad mercantil como sentido común y criterio dominante}

Considero que las respuestas a estas preguntas no son triviales ni simples. Sin embargo, desde el "sentido común" dominante en nuestras sociedades, usualmente se responde a ellas de manera sencilla y taxativa: si las ventas permiten cubrir los costos y obtener una diferencia de dinero significativa al final del proceso, entonces el negocio es viable; si no se alcanzan a cubrir todos los costos, o lo que queda no alcanza para generar un ingreso suficiente para todos los participantes, entonces el emprendimiento no resulta viable. En estos casos, la experiencia se debería discontinuar, no tendría sentido sostenerla en el tiempo.

Podemos denominar como autosostenibilidad mercantil, a esta manera simplificada de considerar esta realidad, que plantea que cada emprendimiento debería obtener por su cuenta en el mercado todos los ingresos que requiere para cubrir sus costos y generar un excedente para distribuir entre sus integrantes; sólo en ese caso un emprendimiento podría ser viable o sostenible. Nuestra intención en el presente trabajo es complejizar esta mirada, incorporando otras perspectivas y criterios de análisis.

De todas maneras, partimos de la base que la perspectiva que acabamos de sintetizar es la más usual entre los propios trabajadores autogestionados, que se enfrentan en la práctica con las dificultades que les presenta la realidad del mercado y no encuentran suficientes herramientas para resolverlas por su propia cuenta.

También es la mirada dominante en la mayor parte de las políticas de promoción de este tipo de emprendimientos, tanto de las estatales como de las que se impulsan desde las organizaciones de la sociedad civil. Estas intervenciones suelen ofrecer apoyos iniciales para poner en funcionamiento o darles impulso a experiencias recién iniciadas, pero luego se espera que -en un corto o mediano plazo- los propios emprendimientos empiecen a ser "autosuficientes" y dejen de necesitar subsidios o ayudas externas.

Y es, también, lo que piensa la gran mayoría de la gente común: a estos emprendimientos les tienen que "cerrar los números" sin apoyo externo. Y desde ese punto estamos a un paso de la valoración negativa que muchos sobre las políticas de promoción de emprendimientos y sus destinatarios, que se 
expresa en una frase que más de una vez he escuchado: "si no son capaces de desarrollar un negocio rentable, que se dediquen a otra cosa; no tenemos que seguir manteniendo vagos con el dinero de todos".

\section{I.3. Nuevas formas de trabajo emergentes y socialmente necesarias}

Ahora bien, nos proponemos reflexionar un poco más sobre este punto: los trabajadores autogestionados (organizados en cooperativas, asociaciones, microemprendimientos, etc.), luego de sufrir exclusiones varias (educativas, laborales y materiales de todo tipo), están abriendo nuevos caminos en nuestra sociedad. Además de producir bienes y servicios que son útiles para resolver necesidades (alimenticias, de vestido, de hábitat, etc.), generalmente están produciendo otros beneficios sociales, como la inclusión laboral y social de grupos sociales relativamente excluidos o vulnerados en sus derechos (desempleados, mujeres jefas de hogar, jóvenes sin experiencia laboral previa, personas con discapacidad, etc.). Están generando experiencias de formación de una nueva cultura de trabajo asociativa y autogestionada, así como de participación, solidaridad y democracia desde las prácticas concretas.

¿No es ilógico, entonces, que la sostenibilidad de este tipo de emprendimientos de trabajadores autogestionados se termine jugando exclusivamente en base a criterios de éxito mercantil? ¿No son las mismas lógicas y reglas de juego del mercado las que excluyen masivamente del empleo decente y del acceso a los bienes y servicios básicos? Si el sentido último de su emprendimiento es la satisfacción de las necesidades básicas de sus miembros y no la acumulación de ganancias y capital, ¿̇tiene sentido que compitan de igual a igual con grandes empresas capitalistas que se manejan con otra lógica y no producen similares beneficios sociales (externalidades positivas) en su accionar? ¿La racionalidad dominante en la competencia mercantil es compatible con la que orienta un emprendimiento asociativo de trabajadores autogestionados? Por otro lado, ¿son las empresas capitalistas realmente autosuficientes? ¿acaso no reciben una gran cantidad de subsidios y ayudas externas, como pudo apreciarse en los salvatajes estatales a grandes bancos y empresas multinacionales en el marco de la última crisis financiera mundial? Y el sistema educativo, y el sistema de leyes e instituciones vigente ¿no subsidia y protege a las empresas capitalistas?

En fin, es el sentido común de la época con respecto a este tema lo que debe ponerse en discusión. No se trata de una discusión estéril ni meramente teórica, sino que se trata de enriquecer la mirada con la que actuamos como 
sociedad en relación con las nuevas formas de trabajo que están emergiendo a raíz de la crisis del capitalismo y del trabajo asalariado, y que van a ser cada vez más frecuentes en las próximas décadas.

¿Por qué afirmamos que estamos en presencia de una nueva forma de trabajo? No es la primera vez en la historia que se desarrollan formas asociativas y autogestionadas para organizar la producción y el consumo. Pero en la actualidad están emergiendo como alternativa frente a la idea y la experiencia del trabajo asalariado y dependiente, dominante en nuestra sociedad desde hace muchas décadas. ¿Cuál es "la idea de trabajo" con la que se forman los estudiantes? ¿Y cuando los adultos afirman que "falta trabajo" a qué se refieren? Están pensando en el empleo bajo relación de dependencia de un patrón, con un jefe, un horario preestablecido, un sueldo fijo y si fuera posible, con cierta estabilidad, cobertura de salud, vacaciones pagas...

Creemos que no existe aún en la actualidad una cultura del trabajo asociativo y autogestionado extendida entre el conjunto de los trabajadores, que en su mayoría no consideran a ese tipo de actividad como un trabajo posible o deseable. Más de una vez hemos escuchado de boca de miembros de emprendimientos autogestionados frases como: "dejé un tiempo el emprendimiento porque conseguí trabajo", refiriéndose a un empleo dependiente, aunque fuera por poco tiempo y por poco dinero, pero en mayor sintonía con sus expectativas acerca de lo que es "tener un trabajo".

Por todo esto, consideramos que es necesario impulsar el desarrollo de una nueva idea y cultura del trabajo que incluya a las prácticas asociativas y autogestionadas como posibilidad, y que se apoye en los conocimientos y saberes adecuados para llevarlas adelante con eficacia. Pero va a llevar un largo tiempo para que esta nueva cultura se desarrolle... y no depende de lo que pueda hacer cada emprendimiento o grupo de trabajadores en particular, sino de lo que se haga a nivel de toda la sociedad, desde lo educativo, lo económico y lo político. Se trata de una cultura que se irá construyendo a partir de la práctica y la reflexión colectiva, y es un proceso que necesita ser apoyado desde el estado y las políticas públicas.

Entonces, si consideramos -conceptual y políticamente- que los emprendimientos asociativos de trabajadores autogestionados son organizaciones socialmente necesarias para que la totalidad de la población trabajadora tenga la posibilidad de acceder al trabajo y a la satisfacción de sus necesidades, entonces el análisis de la realidad nos muestra que deben contemplarse otros criterios de 
viabilidad o sostenibilidad más amplios que el de la autosostenibilidad mercantil. Fundamentalmente, se debe considerar un claro papel promotor y protector por parte del estado para con este tipo de organizaciones, y que se complementen los recursos que estos emprendimientos obtienen en el mercado con otros recursos y con acciones e instituciones de nivel meso y macro que les permitan ser experiencias viables.

\section{Parte II. Un par de aclaraciones conceptuales}

\section{II.1. Emprendimientos Asociativos de Trabajadores Autogestionados}

Los procesos y prácticas de asociativismo y autogestión entre trabajadores dan lugar a la conformación de organizaciones basadas en esta forma de trabajo, nuestro objeto de estudio, que hemos denominado Emprendimientos Asociativos de Trabajadores Autogestionados (EATA), al que ya nos hemos referido en la parte I y cuya conceptualización profundizaremos en esta sección. ${ }^{3}$

Entendemos que los EATA son organizaciones que presentan los siguientes rasgos característicos:

1. Son emprendimientos asociativos, porque agrupan a dos o más trabajadores provenientes de distintas unidades domésticas (pueden ser familiares entre sí, pero no comparten un mismo presupuesto para atender sus gastos básicos) que se unen voluntariamente para llevar adelante -de manera coordinada y sistemática, aunque bajo diversas formas organizativas, inscriptas legalmente o no- actividades que les permitan alcanzar objetivos comunes, vinculados a la reproducción de sus condiciones materiales de existencia.

2. Son emprendimientos de trabajadores y centrados en el trabajo humano, que no surgen a partir de la existencia de un capital que busca ser valorizado, sino que se originan y despliegan a partir de las capacidades de trabajo de sus integrantes -recurso central del emprendimiento- que ellos mismos organizan y gestionan -así como a los demás recursos disponibles, factores no centrales sino de apoyo- en función de sus propios intereses, siendo la reproducción de la vida de los trabajadores no el medio, sino el fin.

3 Recupero aquí un desarrollo conceptual realizado en el marco de mi tesis de maestría (Vázquez, 2010a) 
3. Son emprendimientos autogestionados, ya que no existe en ellos un dueño o un patrón, sino que el conjunto de los trabajadores que los integran son colectivamente propietarios y/o poseedores de los medios de producción, y quienes se organizan y toman decisiones -de acuerdo con ciertas reglas explícitas o implícitas que ellos mismos se dan-bajo formas democráticas y participativas.

4. Se trata de emprendimientos que llevan adelante actividades de producción de bienes y servicios, principalmente destinados para la venta en los mercados y la generación de ingresos monetarios, si bien es posible que una parte significativa de la producción sea distribuida directamente entre los trabajadores y destinada al autoconsumo, o bien al intercambio en mercados solidarios con moneda social.

5. En estas organizaciones, las prácticas y relaciones sociales se apoyan en el reconocimiento de valores tales como solidaridad, confianza y pluralismo, tanto hacia adentro del grupo de trabajadores (lo que se manifiesta, por ejemplo, en la distribución con tendencia igualitaria del ingreso neto producido), como hacia afuera, en las relaciones con la comunidad de la que forman parte.

6. Finalmente, los EATA son organizaciones económicas cuyo objetivo y sentido último es la reproducción de la vida de los trabajadores y sus familias, estando orientados hacia la satisfacción de sus necesidades y no hacia la acumulación de ganancias y capital.

Este conjunto de rasgos construyen un "modelo o tipo ideal" de EATA. Consideramos que este tipo de emprendimientos ${ }^{4}$ presentan en la realidad las características recién planteadas, aunque asumimos que no todos los rasgos se dan de la misma forma y en la misma intensidad en todos los EATA, sino que se manifiestan en las prácticas de manera gradual y tendencial (Gaiger, 2004b).

Cabe reconocer que en nuestros países y dentro del campo de la ESS, se han venido planteando una variedad de conceptualizaciones que intentan dar cuenta de este tipo de organizaciones. Entre ellas,

4 Como ya se dijo, dentro de este recorte quedan comprendidos: emprendimientos barriales que agrupan a familiares y vecinos para la producción de bienes (alimentos, textiles, artesanías, vinculados a la vivienda, etc.); organizaciones de productores locales que se juntan para financiarse, comprar, producir y/o vender colectivamente; empresas recuperadas y otras cooperativas de trabajo que agrupan a gran cantidad de trabajadores; entre otras iniciativas. 
destacamos a las siguientes: Organizaciones Económicas Populares ${ }^{5}$ (Razeto, 1990), Emprendimientos Económicos Solidarios ${ }^{6}$ (Gaiger, 2004b) y Emprendimientos Asociativos Mercantiles ${ }^{7}$ (Coraggio y Federico Sabaté, 2010).

Entre otros aspectos, en nuestra conceptualización de los EATA nos interesa remarcar que se trata de emprendimientos de trabajadores (preferimos no llamarlos emprendedores), no necesariamente de sectores pobres, y de emprendimientos cuya racionalidad está estrechamente vinculada a la lógica económica de las unidades domésticas de sus integrantes, esto es la reproducción ampliada de la vida de sus miembros (Coraggio, 2010).

\section{II.2. Breve disgresión terminológica: ¿viabilidad o sostenibilidad?}

En este punto quisiera plantear una breve disgresión acerca de los términos "viabilidad" y "sostenibilidad", que venimos utilizando indistintamente en nuestro texto. ¿Qué entendemos por viable? ¿Y por sostenible? ¿Cuál es la palabra más adecuada para utilizar en relación a los

5 Luis Razeto define a las Organizaciones Económicas Populares como pequeños grupos que se organizan en conjunto y solidariamente para encarar sus necesidades económicas, sociales y culturales más inmediatas. Sus características distintivas son: "se desarrollan en los sectores populares, entre los más pobres y marginados"; "tienen objetivos precisos y organizan racionalmente los recursos y medios para lograrlos"; "implican relaciones y valores solidarios"; "quieren ser participativas, democráticas, autogestionarias y autónomas"; "combinan sus actividades económicas con otras sociales y de acción política"; "pretenden ser distintas y alternativas respecto de las formas organizativas capitalistas y aportar a un cambio social en la perspectiva de una sociedad más justa”. (Razeto, 1990:127-129; 1997:34-36)

6 "Los Emprendimientos Económicos Solidarios abarcan diversas modalidades de organización económica, originadas en la libre asociación de los trabajadores, con base en principios de autogestión, cooperación, eficiencia y viabilidad. Agrupando a individuos excluidos del mercado de trabajo, o motivados por la fuerza de sus convicciones, y en búsqueda de alternativas colectivas de supervivencia, los EES llevan a cabo actividades de producción de bienes o servicios, comercialización y crédito. Se presentan en forma de emprendimientos productivos, cooperativas y empresas de autogestión y combinan sus actividades económicas con acciones de índole educativa y cultural, valorando el sentido de la comunidad de trabajo y el compromiso con la comunidad local en la que están insertos." (Gaiger, 2004b:229)

7 "Los Emprendimientos Asociativos Mercantiles agrupan trabajadores pertenecientes a distintas unidades domésticas que desarrollan conjuntamente actividades de producción y/o venta de bienes y servicios en el mercado. Sus actividades están dirigidas a generar ingresos monetarios, pero sus fines no son los de acumulación privada de capital. Los ingresos obtenidos se destinan a cubrir los costos de producción, a distribuir entre los trabajadores para atender necesidades mediante el consumo, o para sostener otros emprendimientos sociales o actividades comunitarias." (Coraggio y Federico Sabaté, 2010:20) 
emprendimientos asociativos autogestionados por sus trabajadores?

Debo confesar que hasta el momento sentía un rechazo con respecto a usar el término "viabilidad" para este tipo de experiencias, tal vez debido al uso extendido de esta palabra en los llamados "estudios de viabilidad" utilizados para predecir el éxito o fracaso de un proyecto de inversión a partir de flujo de ingresos y egresos esperados en el futuro, asumiendo una perspectiva capitalista. En nuestra opinión, las iniciativas autogestionadas por trabajadores no pueden ser correctamente evaluadas desde esa perspectiva "inversora", y el éxito o fracaso de un proyecto de este tipo es el resultado de un proceso bastante más complejo e impredecible antes de iniciarse la experiencia.

Pero buscando en la etimología de la palabra, pude encontrar ciertas dimensiones que me han ayudado a "refrescar" el concepto: la palabra "viabilidad" surge de la conjunción de dos vocablos latinos: vita, que puede traducirse como "vida", y bilis, que es equivalente a "posibilidad". La pregunta por la posibilidad (y no la certeza) de que una experiencia pueda tener vida (o no), me resulta un punto de partida más interesante.

Yotro significado de la palabra me ha aportado otra razón para "amigarme" con el término "viable" en relación con los emprendimientos de trabajadores: según el diccionario de la Real Academia Española esta palabra hace referencia a la condición del "camino o vía donde se puede transitar". Me gusta asimilar estas experiencias a caminos que se abren y que no se pueden conocer del todo hasta que se recorren, y que los obstáculos que van apareciendo le van dando forma al propio camino... ${ }^{8}$

El término "sostenibilidad" es hasta el momento el más utilizado en los análisis y debates desarrollados desde el campo de la Economía Social y Solidaria latinoamericana en los últimos años (Coraggio, 2008 y 2009; Gaiger 2008), así como en nuestros propios trabajos previos (Vázquez, 2010a y 2010b).

Si recurrimos nuevamente a la definición de la RAE, veremos que "sostenible" refiere a "lo que se puede mantener durante largo tiempo sin agotar los recursos o causar grave daño al medio ambiente". Y en general,

8 Uno de los evaluadores de este artículo me ha señalado acertadamente que debería incorporar en este punto el concepto de "viabilidad sistémica" y la aplicación al campo de las organizaciones de la idea de "sistemas viables". He tomado nota de la crítica y agradezco la sugerencia, pero no estoy en condiciones de incorporar en este breve apartado exploratorio mayores referencias a dicha perspectiva hasta no profundizar mis lecturas al respecto. 
vemos que un buena parte de las veces que se usa este término se lo relaciona directamente con la cuestión ecológica. Ahora bien, aceptando que la ambiental es una dimensión importante para evaluar los emprendimientos de los trabajadores, personalmente no considero que sea la cuestión central en la discusión que estamos queriendo dar.

Otra definición muy extendida (a la que se llega simplemente "googleando" la palabra) es la que considera "sostenible" a algo "que está en condiciones de conservarse o reproducirse por sus propias características, sin apoyo externo ni merma de recursos existentes" (http://definicion.de/ sostenible/). Aquí claramente se está estableciendo una equivalencia entre la idea de sostenibilidad y la de "autosostenibilidad", que es justamente la idea dominante en el sentido común que queremos poner en discusión.

Por lo expuesto, entonces, en adelante nos interesaría reflexionar más desde la idea de lo "viable" que de lo "sostenible". Pero dado que la discusión en el campo de la ESS ya estaba instalada con el segundo término, iremos planteando este cambio por ahora de forma parcial y gradual.

Parte III. El debate académico sobre la sostenibilidad de los emprendimientos asociativos de trabajadores autogestionados desde una perspectiva sustantiva y plural

\section{III.1. La pluralidad de principios e instituciones de la economia sustantiva}

En esta tercera parte del trabajo queremos realizar un aporte a la discusión sobre los criterios de viabilidad y sostenibilidad, en diálogo con otros autores del campo de la Economía Social y Solidaria (ESS) de América Latina. ${ }^{9}$

Para ello, nos apoyamos en la perspectiva de la economía sustantiva, que establece que son diversas (plurales) las formas en las que una sociedad puede organizar (y de hecho, organiza) lo económico. El concepto sustantivo de economía planteado por Karl Polanyi hace referencia al proceso de interacción entre los hombres entre sí y con la naturaleza a partir del cual toda sociedad

9 Este tema lo he desarrollado con mayor profundidad en mi tesis de maestría (Vazquez, 2010a) y luego en un artículo que presenta sus principales conclusiones (Vázquez, 2010b). Invito a los lectores interesados en profundizar en esta discusión a leer esos textos que aquí estamos sintetizando. 
se organiza para proveer las condiciones materiales que permitan satisfacer las necesidades de todos sus miembros. Este proceso resulta organizado y estabilizado en cada sociedad mediante la combinación variable de un conjunto de principios e instituciones que pautan los comportamientos económicos de personas y grupos. (Coraggio, 2009a)

Polanyi postula que en todas las sociedades conocidas se encuentran presentes (al menos) cuatro principios económicos: reciprocidad, redistribución, administración doméstica e intercambio. Cada uno de estos principios se desarrollan en combinación con ciertos modelos institucionales: simetría, centralidad, autarquía y mercado, respectivamente. ${ }^{10}$

Cuando se habla de reciprocidad como principio económico se hace referencia a intercambios entre personas o grupos que se relacionan entre sí desde una posición similar (simétrica) en cuanto al lugar que ocupan en una sociedad (integrantes de una comunidad étnica o territorial, de una asociación o cooperativa, etc.). Es necesario señalar que el intercambio recíproco no implica intercambio de equivalentes, ni simultáneo ni diferido en el tiempo. Es el acto de dar algo al otro, lo que se devuelve, y no el contenido de lo que se da. Por ello, el principio de reciprocidad está estrechamente relacionado con el concepto y la práctica del don. El don -que se da, en principio, libre y gratuitamente- puede crear un vínculo social entre quien da y quien recibe, que sentirá una cierta obligación a devolver.

La redistribución como principio económico hace referencia a un doble flujo de bienes o servicios, que en un primer momento son entregados

10 Así lo plantea el propio Karl Polanyi en La gran transformación, su obra más influyente, publicada originalmente en 1944: "A grandes rasgos, la proposición afirma que todos los sistema económicos conocidos por nosotros hasta el fin del feudalismo en Europa Occidental estuvieron organizados conforme ya con los principios de reciprocidad y redistribución, o economía doméstica, o alguna combinación de los tres. Esos principios fueron institucionalizados con la ayuda de una organización social que (...) hizo uso de modelos de simetría, centricidad y autarquía. En esta estructura, la producción y distribución ordenada de artículos se aseguró mediante una gran variedad de motivos individuales disciplinados por principios generales de conducta. (...) El trueque, permuta o cambio es un principio de conducta económica cuya eficacia depende de la estructura del mercado. A menos que exista tal estructura, siquiera fragmentariamente, la inclinación a efectuar trueques hallará un campo insuficiente: no puede producir precios. Porque así como la reciprocidad es ayudada por una estructura simétrica de organización, y la redistribución es facilitada por cierto grado de centralización, y la economía doméstica debe basarse en la autarquía, de la misma manera la eficacia del sistema de trueque depende de la estructura del mercado. Pero de la misma forma que la reciprocidad, redistribución o economía doméstica puede darse en la sociedad sin prevalecer en ella, el principio de trueque puede también ocupar un lugar subordinado en una sociedad en que otros principios está en ascendencia." (Polanyi, 2011:84, 89) 
desde todos los integrantes de un colectivo o comunidad hacia un centro (representado por una persona o institución, que a su vez suele ejercer cierta autoridad o gobierno dentro del colectivo); y en un segundo momento (no necesariamente inmediato) vuelven a ser distribuidos entre distintos miembros de la comunidad, pero con un criterio diferente al cual fueron recolectados en el movimiento anterior. Acertadamente Polanyi observa que no puede aplicarse el principio de redistribución si no existe una centralidad reconocida por todos los integrantes de un determinado colectivo social.

El principio de administración doméstica "consiste en la producción para el uso propio. Los griegos lo llamaban oeconomĩa, de donde se deriva la palabra economía" (Polanyi, 2011:101). Se trata de la búsqueda y la práctica de producir y almacenar para satisfacer las necesidades de los miembros de un grupo cerrado, que puede ser una familia, una aldea o localidad, una comunidad territorial más amplia, una región o una nación. Se vincula con la autarquía, la autosuficiencia, la capacidad de una unidad de bastarse a sí misma.

La manera en la que estos principios se aplican es diversa y propia de cada sociedad histórica, que los institucionaliza en función de su organización social vigente. Son también diversas las maneras en las que estos principios e instituciones se combinan en cada sociedad, pudiendo ser algunos predominantes y otros subordinados o marginales en una determinada organización social. (Polanyi, 2011)

Enmarcados en esta perspectiva, hemos realizado un análisis sobre las ideas planteadas por diversos autores del campo de la ESS latinoamericana acerca de la sostenibilidad de los emprendimientos asociativos y autogestionarios, y creemos que pueden reconocerse dos grandes líneas de argumentación:

1) las que afirman que, para alcanzar la sostenibilidad, es necesario fortalecer la capacidad de los emprendimientos para competir en los mercados; y

2) las que plantean que la viabilidad de los emprendimientos depende del desarrollo de instituciones y politicas basadas en otros principios económicos: reciprocidad, redistribución y administración doméstica.

Esta diferenciación analítica entre dos conjuntos no debe ocultar el hecho de que todos los autores del campo académico de la ESS reconocen el papel fundamental que debe jugar el estado y sus intervenciones para permitir o favorecer la viabilidad y sostenibilidad de los emprendimientos asociativos de trabajadores autogestionados en el contexto actual de nuestras sociedades capitalistas. 
III.2. Ser sostenibles mejorando la capacidad de competir en los mercados...

Paul Singer (Secretario Nacional de Economía Solidaria del Brasil durante las presidencias de Lula y Dilma) sostiene que las cooperativas de trabajadores autogestionados son una forma de producción superior a la empresa capitalista, y que -si se dan los necesarios apoyos estatales para permitirles una inserción adecuada en los mercados- las cooperativas podrán ser competitivas y conformar un sector integrado y autosostenible, incluso desplazando paulatinamente a las formas capitalistas de producción. Algunos de sus argumentos son los siguientes: los conflictos internos son menores y/o se pueden resolver abierta y participativamente; la información relevante fluye en toda la organización; las decisiones colectivas son más acertadas porque toman en cuenta la opinión y experiencia de todos los trabajadores, entre otras razones. "La extraordinaria variedad de organizaciones que componen el campo de la economía solidaria permite formular la hipótesis de que ella podrá extenderse hacia todos los campos de actividad económica. No hay, en principio, ningún tipo de producción y distribución que no pueda ser organizado como emprendimiento solidario". (Singer, 2007)

El mismo Singer aclara que es necesario que se garanticen ciertas condiciones para lograr este resultado: "para que esta hipótesis se haga realidad en los diversos países es necesario garantizar las bases de sustentación para la economia solidaria. Las más importantes son las fuentes de financiación, redes de comercialización, asesoramiento técnico científico, formación continua de los trabajadores y apoyo institucional y legal de parte de las autoridades gubernamentales." (Singer, 2007:73) Para este autor, así como las empresas capitalistas ya disponen de bases de sustentación (apoyo de otros grupos económicos, bancos comerciales, cadenas de comercialización, etc.), el apoyo financiero, comercial y tecnológico es clave para que los emprendimientos autogestionarios puedan insertarse más competitivamente en los mercados frente a aquellas. Es en este aspecto en donde Singer pone el acento y para lo cual demanda la imprescindible acción promotora por parte del estado. Una vez lograda esta estructura pública de promoción y apoyo -que ofrezca una "base de sustentación"- la condición clave para la sostenibilidad pasaría a ser la capacidad de autogeneración de ingresos por parte de los emprendimientos a partir de su inserción mercantil. 
Por su parte, Luiz Inácio Gaiger (investigador que ha coordinado los estudios más abarcativos sobre emprendimientos de la economía solidaria, en los que se encuestaron más de 22.000 emprendimientos asociativos en distintos estados del Brasil) afirma en varios de sus trabajos que los emprendimientos de la economía solidaria pueden ser considerados organizaciones viables y alternativas sólo si logran aprovechar el potencial productivo del trabajo asociativo y autogestionado, tanto en su organización y relaciones internas, como en sus vinculaciones con actores e instituciones externas. (Gaiger, 2006, 2007 y 2008)

Gaiger impulsa una reflexión sobre el emprendedorismo en el marco del trabajo asociativo y autogestionado, y plantea que debe hacerse desde un abordaje diferente al usual: "Por ser organizaciones económicas los emprendimientos asociativos necesitan de emprendedorismo; por ser intentos alternativos precisan innovar en ese campo a través de un estilo de emprendedorismo propio, de carácter participativo y democrático" (Gaiger, 2008:63). El emprendedorismo, que suele ser considerado como un atributo individual, en los emprendimientos asociativos puede ser un atributo colectivo.

Ahora bien, este investigador plantea la necesidad de promover activamente el desarrollo de esta capacidad emprendedora, porque estima que no se generará espontáneamente. Para ello, Gaiger afirma que es fundamental conocer y saber aprovechar "las virtudes de la comunidad de trabajo", de modo que se pueda plasmar este potencial en resultados concretos. Sintéticamente, sostiene que la cooperación en el trabajo multiplica la capacidad individual de cada trabajador; las decisiones colectivas conducen a resultados más eficientes; compartir conocimientos e información estimula la innovación y reduce costos de transacción; la confianza y el sentido de pertenencia a un proyecto común estimulan y motivan adicionalmente. Es en este sentido, que este autor afirma que "la dimensión emprendedora es indisociable de la dimensión solidaria de los emprendimientos”. (Gaiger, 2008:62)

La posibilidad de que estas organizaciones puedan llegar a ser sostenibles se juega en la capacidad que tenga cada una de sacar provecho del potencial de sus relaciones de trabajo basadas en la cooperación y la solidaridad: "el diferencial decisivo para el éxito de los emprendimientos reposa en su capacidad de conciliar las relaciones de trabajo que le son propias con los imperativos de la eficiencia, de modo de convertir la propia cooperación en la viga-maestra de una nueva racionalidad. En esas circunstancias, los resultados pasan a depender en buena medida de las virtudes del trabajo asociado, en procura 
de la sobrevivencia del emprendimiento y en favor de los propios productores. El solidarismo y la cooperación en el trabajo, una vez internalizados como práctica cotidiana, propiciarían factores adicionales de eficiencia, en beneficio del emprendimiento." (Gaiger, 2006:5).

En síntesis, ambos autores plantean la necesidad de que los emprendimientos de trabajadores autogestionados mejoren sus capacidades productivas y puedan competir con éxito en los mercados. Para ello, afirman que es mucho lo que hay que mejorar dentro de cada emprendimiento, pero no dejan de señalar que el papel del estado es clave, apoyando y promoviendo a estas iniciativas desde el inicio, hasta que tengan capacidad de sostenerse con sus propios ingresos mercantiles.

En un cierto contraste con estos planteos, José Luis Coraggio (argentino, director de la Maestría en Economía Social de la Universidad Nacional General Sarmiento) discute sobre la pertinencia de procurar el éxito mercantil como vía para alcanzar la sostenibilidad de este tipo de experiencias. Por un lado, enfatiza que esa meta no depende solamente de los esfuerzos ni de los resultados de cada uno de dichos emprendimientos y de los trabajadores que los integran. Y considera que no puede evaluarse la sostenibilidad microeconómica de un emprendimiento sin incorporar en la mirada al contexto socioeconómico, cultural y político, o mejor dicho, sin trabajar sobre la construcción y modificación de ese mismo contexto. "En general, planteamos que es ilógico pensar en lograr otra economía por medio del individualismo metodológico (cada emprendimiento debe ser viable, y por agregación toda la economía social resultaría serlo). El cambio del contexto estructural es condición para la viabilidad y sostenimiento de las iniciativas particulares. Sin construcción de un contexto meso-socioeconómico consistente es poco probable sostener los emprendimientos de este nuevo sector." (Coraggio, 2008:52)

Coraggio apunta su crítica a la ideología mercantilista que reduce el problema de la sostenibilidad económica de cada emprendimiento a su capacidad de competir e internalizar "exitosamente" las reglas de juego del mercado, privilegiando como factor determinante la posibilidad de generar un excedente, entendido como saldo monetario favorable entre ingresos y gastos. Por un lado, plantea que si ese saldo monetario favorable del emprendimiento se logra a partir de sacrificar la calidad de vida de sus trabajadores, se está frente a una irracionalidad material. Por otro lado, si lo que se busca es construir "un sector de actividad económica no regido por la acumulación privada de capital sino por la reproducción ampliada de la vida de todos" es 
ilógico que sea la capacidad de competir en los mercados actuales (dominados por la racionalidad capitalista) lo que determine qué producir, cómo hacerlo, quiénes y para quiénes. Desde su punto de vista, algunos argumentos carecen de una mirada suficientemente crítica hacia el mercado capitalista y la lógica que implica: "El tema de la inevitabilidad (si no la deseabilidad) de pasar exitosamente 'la prueba del mercado' es recurrente en el campo de los agentes y promotores de otra economía. No se quiere imponer otra economía, sino ganar la voluntad de los compradores en libre competencia con los productos del capital." (Coraggio, 2007:26)

\section{III.3. Construir viabilidad a partir del desarrollo de instituciones $y$ politicas basadas en otros principios económicos: reciprocidad, redistribución y administración doméstica}

En esta línea, José Luis Coraggio plantea que "para poder aplicar un criterio coherente de sostenibilidad, que contrarreste la idealización de la empresa mercantil en un mercado perfecto, la teoría crítica de la economía social debe desarrollar un criterio de sostenibilidad socioeconómica, que sea el concepto propio de la ESS en un proceso de transición, y que admita la vía de aparentes 'subsidios' económicos generalizados (educación, capacitación, exención de impuestos, sistemas de salud, etc.) a partir del principio de redistribución progresiva por parte de la economía pública, así como aportes de trabajo u otros recursos (trabajo voluntario, redes de ayuda mutua, uso de la vivienda para la producción, etc.) muchas veces basados en relaciones de reciprocidad y no computados como costos." (Coraggio, 2009b:357).

Coraggio afirma que la sostenibilidad de los emprendimientos asociativos dependerá de múltiples y variadas condiciones, incluso de las capacidades de los trabajadores en el nivel micro, pero en mayor medida dependerá de que el estado pueda producir, distribuir y garantizar eficazmente -como derecho a todos los ciudadanos- el acceso desmercantilizado a una cantidad y calidad de bienes públicos, que promuevan: i) en general, la reproducción de la vida de las personas en sociedad: educación, salud, vivienda, seguridad social y personal, acceso a la justicia, política fiscal progresiva y redistributiva, derecho a participar en la gestión de lo público, etc.; y ii) en particular, el desarrollo y consolidación de las formas de producción, distribución y consumo basadas en el trabajo asociativo y autogestionado: acceso al conocimiento científicotecnológico, implementación de normas jurídico-administrativas que las reconozcan y promuevan, canalización del poder de compra del estado hacia este sector, financiamiento adecuado para este tipo de emprendimientos, 
información sobre beneficios y perjuicios sociales o ambientales detrás de cada tipo de producción, regulación social y política de los mercados frente al poder de los monopolios, etc. (Coraggio, 2008 y 2009b)

Sólo dentro de ese marco, se podría esperar (y no en todos los casos ni en todas las circunstancias) que los emprendimientos cubran sus gastos monetarios con los ingresos obtenidos en el mercado. Ya que, desde una perspectiva de economía sustantiva, se afirma que "los emprendimientos económicos pueden no tener beneficios en sentido estricto y sin embargo ser justificables económicamente." (Coraggio, 2009b:358)

La viabilidad de los emprendimientos de la ESS dependerá, entonces: "(a) de las capacidades y disposiciones de los trabajadores que cooperan a nivel micro, (b) de sus disposiciones a cooperar y coordinarse entre unidades microeconómicas (nivel meso), (c) del contexto socioeconómico y cultural (distribución y organización de recursos, funcionamiento de los mercados, definición de necesidades legítimas), y (d) de la existencia de una política de estado conducente" (Coraggio, 2008:46). Las dos primeras condiciones implican un desarrollo de las capacidades emprendedoras enfatizadas en el apartado anterior. Las dos últimas condiciones dependen de la correlación de fuerzas políticas y sociales existente en nuestras sociedades, y por ende de la capacidad de lucha cultural y política de los trabajadores y de un conjunto de movimientos sociales y de las alianzas que se puedan construir.

Otra dimensión muy importante que considera Coraggio es la de los tiempos: la construcción de capacidades, el desarrollo de productos, la adopción de técnicas adecuadas, la mejor inserción en los mercados, entre otras capacidades claves a desarrollar desde lo micro para la sostenibilidad de los emprendimientos, son procesos que requieren años de maduración, tiempos mucho mayores que los que algunos programas establecen como deseables. "En tanto la productividad y todas las capacidades que están detrás de ella se aprenden, hace falta tiempo para que los nuevos emprendimientos la adquieran, y esto justificaría un período de incubación subsidiada" (Coraggio, 2008:53). En términos de Gaiger, desarrollar el emprendedorismo necesario requiere de tiempos largos de aprendizaje en un contex to protegido. Las transformaciones sociales, culturales y políticas de nivel meso y macro, probablemente sean aún más prolongadas, "pues se trata de cambios institucionales, culturales, de relaciones de poder, de constitución de nuevos sujetos colectivos, de reformas profundas del Estado y de la cultura política" (Coraggio, 2008:56). Este razonamiento indicaría que si en el corto plazo los emprendimientos 
no lograran ingresos mayores a sus costos (como de hecho sucede), entonces las políticas de promoción deberían contemplar mayores apoyos y subsidios, sostenidos a lo largo del tiempo.

Otros aportes a tener en cuenta en esta línea son los que proponen institucionalizar estrategias basadas en el principio de la administración doméstica y en el modelo de la autarquía, focalizando menos en la competitividad de los emprendimientos frente a las empresas de capital, y más en la construcción de sistemas locales relativamente autárquicos de actividades productivas articuladas, orientadas hacia la satisfacción de las necesidades de las comunidades. Uno de los autores que más claramente propone esta alternativa es Franz Hinkelammert, quien afirma que "un desarrollo generalizado solamente es posible interviniendo en los mercados, de manera que quien pierde en la competencia no sea condenado a muerte. Por esta razón, el perdedor de la competencia tiene el derecho de protegerse. Pero no sólo el derecho. También es económicamente racional que lo haga. (...) Pero eso presupone un proteccionismo nuevo, diferente del clásico. Tiene que tener lugar dentro de la sociedad y no simplemente en sus fronteras políticas externas. Tiene que permitir y fomentar sistemas locales y regionales de división del trabajo, que en lo posible estén desconectados de la competencia de las empresas capitalistas orientadas por la acumulación de capital. Eso puede tener las más variadas formas: desde la protección de formas tradicionales de producir que todavía hoy sobreviven (...), hasta la reconstitución de formas de producción simple de mercancía en los sectores urbanos... Hoy la sobrevivencia de la mayoría de la población mundial solamente es posible si sobrevive en producciones nocompetitivas en el marco de una competencia globalizada" (Hinkelammert, 1999:11-12)

Por su parte, Susana Hintze considera necesario el desarrollo actual de un sistema público reproducción del trabajo asociativo y autogestionado. Así como el Estado de Bienestar de posguerra construyó un sistema público de reproducción de la fuerza de trabajo complementario al salario (Topalov, 1979), Hintze plantea la necesidad de que el estado intervenga decisivamente en el momento de construir un nuevo sector de economía que favorezca la reproducción de la sociedad en tiempos de crisis. Y que esa intervención no debería operar sólo a nivel micro, promoviendo que los emprendimientos puedan acceder al mercado, sino que la principal condición de sostenibilidad de un nuevo sector de ESS sería que el estado garantizara la reproducción de la vida de los trabajadores y de las organizaciones que están experimentando en la construcción de estas nuevas formas de hacer economía. Es decir, que para 
pensar la sostenibilidad siempre deben tenerse en cuenta dos planos: el de la reproducción de las organizaciones de la ESS (los emprendimientos); y el de la reproducción de los sujetos que trabajan en dichas organizaciones. (Hintze, 2009)

La propuesta de Hintze es reformar y reorientar los sistemas y politicas de seguridad social de manera tal que incluyan y protejan a los trabajadores autogestionados mediante esquemas redistributivos, y no sólo a los asalariados formales mediante esquemas contributivos. El desafío central que plantea Claudia Danani va en la misma dirección: "En el siglo XXI la seguridad social enfrenta un reto principal que atraviesa todas sus perspectivas: el de proveer garantías y certidumbres a los más amplios sectores de la población, en condiciones de accesibilidad y calidad compatibles con la capacidad de satisfacción de necesidades de las sociedades modernas. En este aspecto, la seguridad social constituye un problema central para la economía social y solidaria, como conjunto de prácticas que pugnan por una economía institucionalizada de manera más solidaria, con predominio de los principios de reciprocidad y redistribución progresiva por sobre el de mercado" (Danani, 2009:336). Por supuesto, estas políticas sólo podrán llevarse a cabo si el conjunto de los trabajadores y sus organizaciones logran construir una fuerza social y política suficientemente potente en esta dirección, lo que refuerza la idea de que la búsqueda de la sostenibilidad tiene un alto componente de lucha cultural y política contrahegemónica.

Un último aporte que nos gustaría rescatar es de Lia Tiriba, quien enfatiza en los condicionantes culturales para la sostenibilidad de los emprendimientos de la ESS, y en las necesidades vinculadas a la formación de los sujetos para su construcción. Ella advierte que el desarrollo de una nueva cultura del trabajo (asociativo y autogestionado) requiere tiempos y recursos amplios, y por eso este proceso queda directamente vinculado con la posibilidad de la acción estatal en este campo. (Tiriba, 2007)

Con respecto al proceso más específico de transformación cultural, Tiriba subraya la importancia de los procesos pedagógicos y el aprendizaje a partir de la práctica productiva y participativa, para la gradual apropiación por parte de los trabajadores de los conocimientos necesarios para el desarrollo y el fortalecimiento de sus emprendimientos. Ahora bien, este espacio de producción de saberes en el trabajo, debe entenderse en un sentido más amplio: una nueva cultura del trabajo "no se produce solamente a partir del espacio de la producción, sino también en los diversos espacios/redes que constituyen 
al sujeto" (Tiriba, 2007:201). En esa línea, plantea la necesidad de integrar, redireccionar transformar el sistema educativo formal en función de esta propuesta, así como fortalecer y articular los procesos de educación popular y formación continua existentes. (Tiriba, 2000)

\section{Palabras finales: hacia una perspectiva plural de la viabilidad de las experiencias de trabajo asociativo y autogestionado}

Aliniciar este trabajo nos preguntábamos por la viabilidad y sostenibilidad de los emprendimientos asociativos de trabajadores autogestionados, partiendo de sus dificultades para competir exitosamente en mercados dominados por grandes empresas capitalistas.

Hemos planteado que las respuestas desde el "sentido común" condicionan la sostenibilidad al éxito en el mercado de cada experiencia en el corto plazo. Caracterizamos a dicha perspectiva hegemónica como de autosostenibilidad micro y mercantil, y argumentamos que esa mirada puede y debe ser problematizada y complejizada, sobre todo considerando la relevancia social de estas experiencias impulsadas por los trabajadores en un contexto de crisis del empleo asalariado y del capitalismo como sistema global, en donde se revela que ni siquiera las grandes empresas multinacionales se "autosostienen" sino que dependen del apoyo y subsidio estatal.

Creemos que los interrogantes que planteamos en la primera parte del texto encuentran respuestas posibles en los argumentos presentados en el resto del artículo, especialmente en la parte III. Allí hemos analizado diversos aportes de diferentes autores del campo académico de la Economía Social y Solidaria de América Latina sobre las condiciones de viabilidad y sostenibilidad de las experiencias de trabajo asociativo y autogestionado. Habiendo organizado este debate en función de los principios económicos plurales postulados por la perspectiva de la economía sustantiva, consideramos tener argumentos suficientes para confrontar con el concepto de autosostenibilidad y pensar esta problemática desde una mirada de viabilidad o sostenibilidad plural.

Esto implica reconocer la pluralidad de principios (reciprocidad, redistribución, administración doméstica e intercambio mercantil), la pluralidad de niveles (micro, meso y macro) y de dimensiones (social, económica, cultural y política) que hacen a la viabilidad y sostenibilidad de los emprendimientos asociativos de trabajadores autogestionados. Esta 
perspectiva plural se puede traducir en la práctica en un conjunto complejo de estrategias, políticas y recursos que confluyen hacia la reproducción de estas organizaciones y de los trabajadores que las integran.

No negamos la necesidad de desarrollar las capacidades emprendedoras de estas organizaciones, y así mejorar su capacidad de producir y competir con mayor calidad y eficiencia en los mercados. Pero creemos que su viabilidad se juega también en buena medida en la capacidad de construir relaciones de fuerza favorables para impulsar políticas de promoción y protección de las experiencias asociativas y autogestionadas, basadas en otros principios e instituciones.

En un plano más concreto, y a modo de ejemplo de lo que estamos planteando, se discute mucho (en Argentina, lo mismo sucede en otros países de nuestra región) acerca de la legitimidad del subsidio estatal a los emprendimientos asociativos autogestionados por sus trabajadores. En nuestro país, en los últimos años se establecieron políticas públicas de promoción que subsidian proyectos aportando máquinas e insumos para comenzar la producción. Nuestra postura es que estos subsidios además de legítimos y necesarios, resultan insuficientes. Muchas necesidades de estos emprendimientos no pueden ser cubiertos con esos pequeños subsidios ni con sus ingresos mercantiles en el corto plazo, a saber: sostener un espacio e instalaciones adecuadas en donde desarrollar las actividades productivas y/o comerciales, los medios de transporte para el traslado de las materias primas y de los productos terminados desde y hacia los mercados, los conocimientos y medios tecnológicos que les permitan producir con mayor calidad y capacidad de competir, los costos vinculados a la publicidad y comercialización de sus productos, etc. Desde nuestra perspectiva, los subsidios públicos ${ }^{11}$ (basados en el principio de redistribución) deberian ser más amplios en su cobertura y sostenidos en el tiempo, porque consideramos que es el conjunto de la sociedad a través del estado quien debe sostener la búsqueda que encarnan estas experiencias emergentes, así como garantizar el derecho al trabajo y la satisfacción de las necesidades básicas de sus integrantes. Porque estos emprendimientos cumplen -como ya dijimos- un importante rol social, al integrar a trabajadores excluidos del empleo asalariado, y al sentar las bases para el impulso de otras formas de

11 Este apoyo estatal puede materializarse de maneras diversas, no solamente con subsidios monetarios a los trabajadores. Por ejemplo, puede establecerse (como ya ocurre en algunos países europeos) que los emprendimientos de la ESS tengan prioridad sobre las empresas capitalistas para ser proveedores del Estado, y que de esa manera se destine una proporción significativa de las compras públicas hacia este sector, garantizando un piso de demanda y de ingresos por ventas en estas organizaciones. 
trabajo, de otra economía en la que todos puedan vivir dignamente.

Espero que estas reflexiones que hemos compartido puedan resultar de utilidad para repensar esta problemática y las formas de intervenir frente a ella. Quedamos a disposición para seguir intercambiando ideas y experiencias en ese sentido.

\section{Referencias bibliiográficas}

Abramovich, Ana Luz y Vázquez, Gonzalo (2005) "Reflexiones sobre las formas de promoción y apoyo a emprendimientos productivos" en Revista Medio Ambiente y Urbanización no 61, IIED-AL, Buenos Aires, Febrero de 2005 .

Coraggio, J.L. y Federico Sabaté, A. (dir.) (2010): "Emprendimientos socioeconómicos asociativos: su vulnerabilidad y sostenibilidad". Ediciones UNGS, Universidad Nacional de General Sarmiento, Los Polvorines, Argentina.

(2009a): "Polanyi y la

Economía Social y Solidaria en América Latina" en Coraggio, J. L. (org.) ¿Qué es lo económico? Materiales para un debate necesario contra el fatalismo. CICCUS, Buenos Aires.

(2009b): "Sostenibilidad" en Cattani, A., Coraggio, J.L. y Laville, J.L. (organizadores) Diccionario de la otra economía, Colección lecturas sobre Economía Social, UNGS-AltamiraClacso, Buenos Aires.

(2008): "La sostenibilidad de los emprendimientos de la economía social y solidaria". En Revista Otra Economía n³, http://revistas.unisinos.br/index.php/otraeconomia

Danani, Claudia (2009): "Seguridad Social" en Cattani, A., Coraggio, J.L. y Laville, J.L. (organizadores) Diccionario de la otra economia, Colección lecturas sobre Economía Social, UNGS-Altamira-Clacso, Buenos Aires.

Gaiger, Luiz Inacio (2008): "A dimensão empreendedora da economia solidária: notas para um debate necessário”. En Revista Otra Economía n³, http://revistas.unisinos.br/index.php/otraeconomia. 
(2007): "A outra racionalidade da economia solidária. Conclusões do primeiro Mapeamento Nacional no Brasil”, Revista Crítica de Ciências Sociais, 79, Dezembro 2007: 57-77.

(2006): "A racionalidade dos formatos produtivos autogestionários." Sociedade e Estado. Brasília: UNB, v. 21, n. 2, 2006. p. $513-545$.

(2004a). "As emancipaçoes no presente e no futuro". En Sentido e experiencias da economía solidaria no Brasil. Editora da UFRGS, Porto Alegre.

(2004b). "Emprendimientos económicos solidarios". En Cattani, Antonio David, La otra economía, Colección lecturas sobre Economía Social, Altamira, Buenos Aires.

Hinkelammert, Franz (1999): “El huracán de la globalización”, DEI, San José, Costa Rica.

Hintze, Susana (2010): "La política es un arma cargada de futuro: la economía social y solidaria en Brasil y Venezuela”. CICCUS-CLACSO, Buenos Aires.

Polangy, Karl (2011): “La gran transformación. Los orígenes políticos y económicos de nuestro tiempo", Fondo de Cultura Económica, Buenos Aires. Fecha de publicación original de la robra: 1944.

Razeto, Luis (1997): “Los caminos de la economía de solidaridad”. Editorial Lumen Humanitas. Buenos Aires.

(1990): "Economía de Solidaridad y Organización Popular", en "Organizaciones Económicas Populares. Más allá de la informalidad", Servicio Cristiano de Cooperación, Buenos Aires.

Singer, Paul (2007): "Economía Solidaria: un modo de producción y distribución”, en CORAGGIO, J. L. (org.) La economía social desde la periferia: contribuciones latinoamericanas. UNGS/Editorial Altamira, Buenos Aires.

Tiriba, Lia (2007): "Pedagogía (s) de la producción asociada: ¿hacia dónde camina la economía popular?”. En Coraggio, José Luis (org.) La economía social desde la periferia. Contribuciones latinoamericanas. UNGS/Editorial Altamira, Buenos Aires. 
(2000): “Economía popular y movimientos populares (y una vez más, el trabajo como principio educativo)”, Mimeo.

Topalov, Christian (1979): "La urbanización capitalista”. Editorial Edicol, México.

Vázquez, Gonzalo (2010a): “La sostenibilidad de los emprendimientos asociativos de trabajadores autogestionados. Perspectivas y aportes conceptuales desde América Latina”, Tesis de Maestría, Universidad Nacional de General Sarmiento, Los Polvorines, Argentina. Disponible en http:// www. ungs.edu.ar/cm/uploaded_files/pos_tesis/104_tesisGonzalo Vazquez - version final.pdf

(2010b): "El debate sobre la sostenibilidad de los emprendimientos asociativos de trabajadores autogestionados". Publicado en Revista de Ciencias Sociales, segunda época, editada por la Universidad Nacional de Quilmes. Año 2, No 18, primavera de 2010. Disponible en https://www.academia.edu/12494889/El_debate_sobre_la_sostenibilidad_de_los_ emprendimientos_asociativos_de_trabajadores_autogestionados 